\title{
CONVERGENCE AND PERIODICITY OF SOLUTIONS FOR A CLASS OF DIFFERENCE SYSTEMS
}

HONGHUA BIN, LIHONG HUANG, AND GUANG ZHANG

Received 16 January 2006; Revised 27 July 2006; Accepted 28 July 2006

A class of difference systems of artificial neural network with two neurons is considered. Using iterative technique, the sufficient conditions for convergence and periodicity of solutions are obtained in several cases.

Copyright (c) 2006 Honghua Bin et al. This is an open access article distributed under the Creative Commons Attribution License, which permits unrestricted use, distribution, and reproduction in any medium, provided the original work is properly cited.

\section{Introduction}

Consider the following difference system of the form:

$$
\begin{aligned}
& x_{n+1}=\lambda x_{n}+f\left(y_{n}\right), \\
& y_{n+1}=\lambda y_{n}+f\left(x_{n}\right),
\end{aligned}
$$

where $\lambda \in(0,1)$ is a constant, for any $a, b \in \mathbb{R}, f: \mathbb{R} \rightarrow \mathbb{R}$ is given by

$$
f(u)= \begin{cases}1, & u \in[a, b] \\ 0, & u \notin[a, b] .\end{cases}
$$

The system (1.1) can be viewed as the discrete version of the following two-neuron network model:

$$
\begin{aligned}
& \frac{d x}{d t}=-\alpha x+\beta f(y([t])), \\
& \frac{d y}{d t}=-\alpha y+\beta f(x([t])),
\end{aligned}
$$

where $[\cdot]$ denotes the greatest integer function, $\alpha>0$ represents the internal decay rate, 
$\beta>0$ measures the synaptic strength, $x(t)$ and $y(t)$ denote the activations of the corresponding neurons, respectively, and $f$ is the activation function defined by (1.2).

In recent years, many research efforts have been made in neural modelling and analysis since one of the neural networks models with electronic circuit implementation was proposed by Hopfield in [6]. System (1.3) describes the evolution of a network of two identical neurons with excitatory interactions, which has found interesting applications in image processing of moving objects and has been investigated in [7].

In fact, we can rewrite system (1.3) as the following form:

$$
\begin{aligned}
& \frac{d}{d t}\left(x(t) e^{\alpha t}\right)=\beta e^{\alpha t} f(y([t])), \\
& \frac{d}{d t}\left(y(t) e^{\alpha t}\right)=\beta e^{\alpha t} f(x([t])) .
\end{aligned}
$$

Let $n$ be a positive integer. We integrate (1.4) from $n$ to $t \in[n, n+1)$ and obtain

$$
\begin{aligned}
& x(t) e^{\alpha t}-x(n) e^{\alpha n}=\frac{\beta}{\alpha}\left(e^{\alpha t}-e^{\alpha n}\right) f(y(n)), \\
& y(t) e^{\alpha t}-y(n) e^{\alpha n}=\frac{\beta}{\alpha}\left(e^{\alpha t}-e^{\alpha n}\right) f(x(n)) .
\end{aligned}
$$

For any nonnegative integer $k$, we denote $x(k)$ and $y(k)$ by $x_{k}$ and $y_{k}$, respectively. Let $t \rightarrow n+1$ in (1.5), then it follows that

$$
\begin{aligned}
& x_{n+1}=\frac{1}{e^{\alpha}} x_{n}+\frac{\beta}{\alpha}\left(1-\frac{1}{e^{\alpha}}\right) f\left(y_{n}\right), \\
& y_{n+1}=\frac{1}{e^{\alpha}} y_{n}+\frac{\beta}{\alpha}\left(1-\frac{1}{e^{\alpha}}\right) f\left(x_{n}\right),
\end{aligned}
$$

In view of system (1.6), we consider the following variables:

$$
\begin{gathered}
f^{*}(u)=f\left(\frac{\beta\left(e^{\alpha}-1\right)}{\alpha e^{\alpha}} u\right), \quad a^{*}=\frac{\alpha e^{\alpha}}{\beta\left(e^{\alpha}-1\right)} a, \quad b^{*}=\frac{\alpha e^{\alpha}}{\beta\left(e^{\alpha}-1\right)} b, \\
x_{n}^{*}=\frac{\alpha e^{\alpha}}{\beta\left(e^{\alpha}-1\right)} x_{n}, \quad y_{n}^{*}=\frac{\alpha e^{\alpha}}{\beta\left(e^{\alpha}-1\right)} y_{n}, \quad n=0,1,2, \ldots,
\end{gathered}
$$

and then drop the $*$ to get

$$
\begin{aligned}
& x_{n+1}=\frac{1}{e^{\alpha}} x_{n}+f\left(y_{n}\right), \\
& y_{n+1}=\frac{1}{e^{\alpha}} y_{n}+f\left(x_{n}\right),
\end{aligned}
$$

Obviously, system (1.8) is a special form of system (1.1) with $\lambda=1 / e^{\alpha}$. Thus, we may say that (1.1) includes the discrete version of an artificial neural network of two neurons with piecewise constant argument. 
On the other hand, the dynamics of the systems (1.1) and (1.3) have been extensively studied in the literature. However, most of the existing results are concentrated on the case where the function $f$ is piecewise linear or a smooth sigmoid, see [2-5] and references therein. Huang and $\mathrm{Wu}$ [7] and Meng et al. [9] studied the dynamics of system (1.3). Yuan et al. [10] considered system (1.1), where the signal function $f$ is of the following piecewise constant McCulloch-Pitts nonlinearity: $f(u)=1$ if $u \leq \sigma, f(u)=-1$ if $u>\sigma$, for some constant $\sigma \in \mathbb{R}$.

The aim of this paper is to investigate the convergence and periodicity of solutions for system (1.1) as $f$ is of the digital nature (1.2), which describes the input-output relation of a neuron.

For simplicity, let $\mathbb{N}$ denote the set of all nonnegative integers, and define $N(m)=$ $\{m, m+1, m+2, \ldots\}, N(m, n)=\{m, m+1, \ldots, n\}$ for any $m, n \in \mathbb{N}$ and $m \leq n$. Moreover, we introduce the following notations:

$$
\begin{array}{cc}
I_{11}=\{(x, y) ; x<a, y<a\}, & I_{12}=\{(x, y) ; x<a, y \in[a, b]\}, \\
I_{13}=\{(x, y) ; x<a, y>b\}, & I_{21}=\{(x, y) ; x \in[a, b], y<a\}, \\
I_{22}=\{(x, y) ; x \in[a, b], y \in[a, b]\}, & I_{23}=\{(x, y) ; x \in[a, b], y>b\}, \\
I_{31}=\{(x, y) ; x>b, y<a\}, & I_{32}=\{(x, y) ; x>b, y \in[a, b]\}, \\
I_{33}=\{(x, y) ; x>b, y>b\}, & \gamma_{k}=\frac{b}{\lambda^{k}}(b>0, k \in \mathbb{N}), \\
\Theta=\bigcup_{k \in \mathbb{N}}\left(\left(\gamma_{k}, \gamma_{k+1}\right] \times\left(\gamma_{k}, \gamma_{k+1}\right]\right), & \Lambda=\bigcup_{k \in \mathbb{N}}\left(\left(\gamma_{k+1},+\infty\right) \times\left(\gamma_{k}, \gamma_{k+1}\right]\right), \\
\Omega=\bigcup_{k \in \mathbb{N}}\left(\left(\gamma_{k}, \gamma_{k+1}\right] \times\left(\gamma_{k+1},+\infty\right)\right) .
\end{array}
$$

Obviously,

$$
\bigcup_{i, j=1}^{3} I_{i j}=\mathbb{R}^{2}, \quad \lim _{k \rightarrow+\infty} \gamma_{k}=+\infty, \quad \Theta \cup \Lambda \cup \Omega=I_{33} .
$$

By a solution of the system (1.1), we mean a sequence $\left\{\left(x_{n}, y_{n}\right)\right\}$ of points in $\mathbb{R}^{2}$ that is defined for all $n \in N(1)$ and satisfies (1.1) for $n \in N(1)$. Clearly, for any $\left(x_{0}, y_{0}\right) \in \mathbb{R}^{2}$, system (1.1) has a unique solution $\left\{\left(x_{n}, y_{n}\right)\right\}$ satisfying the initial condition $\left.\left(x_{n}, y_{n}\right)\right|_{n=0}=$ $\left(x_{0}, y_{0}\right)$.

For the general background of difference equations, one can refer to $[1,8]$.

This paper is divided into three parts. The main results and their proofs will be given in Sections 2 and 3, respectively.

\section{Main results}

Throughout this paper, $\left\{\left(x_{n}, y_{n}\right)\right\}$ denotes the unique solution of the system (1.1) with initial value $\left(x_{0}, y_{0}\right) \in \mathbb{R}^{2}$. 
Proposition 2.1. If either $b<0$ or $a>1 /(1-\lambda)$, then $\left(x_{n}, y_{n}\right) \rightarrow(0,0)$ as $n \rightarrow \infty$.

Remark 2.2. When $0 \leq a<b<1 /(1-\lambda)$, solutions of system (1.1) are convergent and periodic. Moreover, if we restrict $a \leq \lambda b$, then the convergence and periodicity are similar to the case as $a<0<b<1 /(1-\lambda)$. Therefore, applying Proposition 2.1, we only consider the case $a<0<b<1 /(1-\lambda)$ in this paper.

Proposition 2.3. If $a<0<b<1 /(1-\lambda)$, then

(1) $\left(x_{n}, y_{n}\right) \rightarrow(0,1 /(1-\lambda))$ as $n \rightarrow \infty$ if $\left(x_{0}, y_{0}\right) \in I_{23} \cup \Omega$;

(2) $\left(x_{n}, y_{n}\right) \rightarrow(1 /(1-\lambda), 0)$ as $n \rightarrow \infty$ if $\left(x_{0}, y_{0}\right) \in I_{32} \cup \Lambda$.

Remark 2.4. By a simple analysis, if $a<0<b<1 /(1-\lambda)$, we can find that the solution $\left\{\left(x_{n}, y_{n}\right)\right\}$ of system (1.1) with the initial value $\left(x_{0}, y_{0}\right) \in \mathbb{R}^{2}$ will be in the region $I_{23} \cup$ $I_{32} \cup I_{33}$ eventually. Note that $\Theta \cup \Lambda \cup \Omega=I_{33}$, by Proposition 2.3, it remains to consider the initial value $\left(x_{0}, y_{0}\right) \in \Theta$.

Theorem 2.5. For $m \in N(1)$, define

$$
\delta_{m}=\frac{1}{1-\lambda}-\frac{\lambda^{m-1}}{1-\lambda^{m+1}}, \quad \epsilon_{m}=\frac{1}{1-\lambda}-\frac{\lambda^{m}}{1-\lambda^{m+1}}
$$

If $a<0<\lambda /\left(1-\lambda^{2}\right) \leq b<1 /(1-\lambda)$ and $b \in\left[\delta_{m}, \epsilon_{m}\right)$, then the solution $\left\{\left(\bar{x}_{n}, \bar{y}_{n}\right)\right\}$ of system (1.1) with the initial value $\left(\epsilon_{m}, \epsilon_{m}\right)$ is periodic with minimal period $m+1$. Moreover, for any solution $\left\{\left(x_{n}, y_{n}\right)\right\}$ of (1.1) with the initial value $\left(x_{0}, y_{0}\right) \in(b, \lambda b+1] \times(b, \lambda b+1]$, $\lim _{n \rightarrow \infty}\left(x_{n}-\bar{x}_{n}\right)=\lim _{n \rightarrow \infty}\left(y_{n}-\bar{y}_{n}\right)=0$.

Theorem 2.6. For $m \in N(2)$, define

$$
\zeta_{m}=\frac{\lambda^{m}}{1-\lambda^{m+1}}, \quad \eta_{m}=\frac{\lambda^{m-1}}{1-\lambda^{m+1}}
$$

If $a<0<b<\lambda /\left(1-\lambda^{2}\right)$ and $b \in\left[\zeta_{m}, \eta_{m}\right)$, then the solution $\left\{\left(\bar{x}_{n}, \bar{y}_{n}\right)\right\}$ of the system (1.1) with the initial value $\left(\eta_{m}, \eta_{m}\right)$ is periodic with minimal period $m+1$. Moreover, for any solution $\left\{\left(x_{n}, y_{n}\right)\right\}$ of system (1.1) with the initial value $\left(x_{0}, y_{0}\right) \in(b, b / \lambda] \times(b, b / \lambda]$, $\lim _{n \rightarrow \infty}\left(x_{n}-\bar{x}_{n}\right)=\lim _{n \rightarrow \infty}\left(y_{n}-\bar{y}_{n}\right)=0$.

Remark 2.7. By the formulations in Theorems 2.5-2.6, it is easy to see that $\lim _{m \rightarrow \infty} \epsilon_{m}=$ $1 /(1-\lambda)$ and $\lim _{m \rightarrow \infty} \eta_{m}=0$. Moreover, we have

$$
\begin{aligned}
& \frac{\lambda}{1-\lambda^{2}}=\delta_{1}<\epsilon_{1}<\delta_{2}<\epsilon_{2}<\delta_{3}<\cdots<\delta_{m}<\epsilon_{m}<\cdots, \quad m \in N(1), \\
& \frac{\lambda}{1-\lambda^{2}}=\zeta_{1}>\eta_{2}>\zeta_{2}>\cdots>\eta_{m-1}>\zeta_{m-1}>\eta_{m}>\cdots, \quad m \in N(2) .
\end{aligned}
$$

Corresponding to Theorems 2.5-2.6, we have the following two results. 
Theorem 2.8. Let $x^{*}=\left[b-\left(1-\lambda^{m}\right) /(1-\lambda)\right] / \lambda^{m}$, and $a<0<\lambda /\left(1-\lambda^{2}\right) \leq b<1 /(1-\lambda)$. For $m \in N(1)$ and $l \in \mathbb{N}$, define

$$
\begin{gathered}
\theta_{m, l}=\frac{\lambda^{(m+2)(l+2)-2}(1-\lambda)+\left(1-\lambda^{m}\right)\left(1+\lambda^{(m+2)(l+1)-1}\right)}{(1-\lambda)\left(1-\lambda^{(m+2)(l+2)-1}\right)} \\
+\frac{\lambda^{m+1}\left(1-\lambda^{m+1}\right)\left(1-\lambda^{(m+2) l}\right) /\left(1-\lambda^{m+2}\right)}{(1-\lambda)\left(1-\lambda^{(m+2)(l+2)-1}\right)}, \\
\mu_{m, l}=\frac{1-\lambda^{m}+\lambda^{m+1}\left(1-\lambda^{m+1}\right)\left(1-\lambda^{(m+2)(l+1)}\right) /\left(1-\lambda^{m+2}\right)}{(1-\lambda)\left(1-\lambda^{(m+2)(l+2)-1}\right)}, \\
\xi_{m, l}=\frac{\left(1-\lambda^{m}\right)\left(1+\lambda^{m+1}-\lambda^{(m+2)(l+2)-1}\right)+\lambda^{2 m+2}\left(1-\lambda^{m+1}\right)\left(1-\lambda^{(m+2)(l+1)}\right) /\left(1-\lambda^{m+2}\right)}{\left(1-\lambda^{(m+2)(l+2)-1}\right)(1-\lambda)} .
\end{gathered}
$$

(1) If $b \in\left[\theta_{m, l}, \mu_{m, l}\right)$, then there exists $a\left(\bar{x}_{0}, \bar{y}_{0}\right) \in\left(x^{*}, \lambda b+1\right] \times\left(x^{*}, \lambda b+1\right]$ such that the solution $\left\{\left(\bar{x}_{n}, \bar{y}_{n}\right)\right\}$ of system (1.1) with the initial value $\left(\bar{x}_{0}, \bar{y}_{0}\right)$ is periodic with minimal period $(m+2)(l+2)-1$. Moreover, for any solution $\left\{\left(x_{n}, y_{n}\right)\right\}$ of system (1.1) with the initial value $\left(x_{0}, y_{0}\right) \in\left(x^{*}, \lambda b+1\right] \times\left(x^{*}, \lambda b+1\right], \lim _{n \rightarrow \infty}\left(x_{n}-\bar{x}_{n}\right)=$ $\lim _{n \rightarrow \infty}\left(y_{n}-\bar{y}_{n}\right)=0$.

(2) If $b \in\left[\xi_{m, l}, \mu_{m, l}\right)$, then there exists $a\left(\bar{x}_{0}, \bar{y}_{0}\right) \in\left(b, x^{*}\right] \times\left(b, x^{*}\right]$ such that the solution $\left\{\left(\bar{x}_{n}, \bar{y}_{n}\right)\right\}$ of system (1.1) with the initial value $\left(\bar{x}_{0}, \bar{y}_{0}\right)$ is periodic with minimal period $(m+2)(l+2)-1$. Moreover, for any solution $\left\{\left(x_{n}, y_{n}\right)\right\}$ of system (1.1) with the initial value $\left(x_{0}, y_{0}\right) \in\left(b, x^{*}\right] \times\left(b, x^{*}\right], \lim _{n \rightarrow \infty}\left(x_{n}-\bar{x}_{n}\right)=\lim _{n \rightarrow \infty}\left(y_{n}-\bar{y}_{n}\right)=$ 0 .

Theorem 2.9. Let $\bar{x}^{*}=\left(b-\lambda^{m}\right) / \lambda^{m+2}$, and let $a<0<b<\lambda /\left(1-\lambda^{2}\right)$. For $m \in N(2), l \in$ $N(1)$, define

$$
\begin{gathered}
\rho_{m, l}=\frac{\lambda^{m}\left[1+\lambda^{(m+1)(l+1)+1}+\lambda^{m+1}\left(1-\lambda^{(m+1) l}\right) /\left(1-\lambda^{m+1}\right)\right]}{1-\lambda^{(m+1)(l+2)+1}}, \\
\tau_{m, l}=\frac{\lambda^{m}\left[1+\lambda^{m+1}\left(1-\lambda^{(m+1)(l+1)}\right) /\left(1-\lambda^{m+1}\right)\right]}{1-\lambda^{(m+1)(l+2)+1}} \\
\omega_{m, l}=\lambda^{m}+\frac{\lambda^{2 m+2}\left[1+\lambda^{m+1}\left(1-\lambda^{(m+1)(l+1)}\right) /\left(1-\lambda^{m+1}\right)\right]}{1-\lambda^{(m+1)(l+2)+1}}
\end{gathered}
$$

(1) If $b \in\left[\rho_{m, l}, \tau_{m, l}\right)$, then there exists a $\left(\tilde{x}_{0}, \tilde{y}_{0}\right) \in\left(\bar{x}^{*}, b / \lambda\right] \times\left(\bar{x}^{*}, b / \lambda\right]$ such that the solution $\left\{\left(\tilde{x}_{n}, \tilde{y}_{n}\right)\right\}$ of system (1.1) with the initial value $\left(\tilde{x}_{0}, \tilde{y}_{0}\right)$ is periodic with minimal period $(m+1)(l+2)+1$. Moreover, for any solution $\left\{\left(x_{n}, y_{n}\right)\right\}$ of system (1.1) with the initial value $\left(x_{0}, y_{0}\right) \in\left(\bar{x}^{*}, b / \lambda\right] \times\left(\bar{x}^{*}, b / \lambda\right], \lim _{n \rightarrow \infty}\left(x_{n}-\tilde{x}_{n}\right)=$ $\lim _{n \rightarrow \infty}\left(y_{n}-\tilde{y}_{n}\right)=0$.

(2) If $b \in\left[\omega_{m, l}, \tau_{m, l}\right)$, then there exists $a\left(\tilde{x}_{0}, \tilde{y}_{0}\right) \in\left(b, \bar{x}^{*}\right] \times\left(b, \bar{x}^{*}\right]$ such that the solution $\left\{\left(\tilde{x}_{n}, \tilde{y}_{n}\right)\right\}$ of system (1.1) with the initial value $\left(\tilde{x}_{0}, \tilde{y}_{0}\right)$ is periodic with minimal 
period $(m+1)(l+2)+1$. Moreover, for any solution $\left\{\left(x_{n}, y_{n}\right)\right\}$ of system (1.1) with the initial value $\left(x_{0}, y_{0}\right) \in\left(b, \bar{x}^{*}\right] \times\left(b, \bar{x}^{*}\right], \lim _{n \rightarrow \infty}\left(x_{n}-\tilde{x}_{n}\right)=\lim _{n \rightarrow \infty}\left(y_{n}-\tilde{y}_{n}\right)=0$.

Remark 2.10. Obviously, $\left[\theta_{m, l}, \mu_{m, l}\right) \subseteq\left(\epsilon_{m}, \delta_{m+1}\right),\left[\xi_{m, l}, \mu_{m, l}\right) \subseteq\left(\epsilon_{m}, \delta_{m+1}\right),\left[\rho_{m, l}, \tau_{m, l}\right) \subseteq$ $\left(\eta_{m+1}, \zeta_{m}\right),\left[\omega_{m, l}, \tau_{m, l}\right) \subseteq\left(\eta_{m+1}, \zeta_{m}\right)$. Moreover,

$$
\begin{gathered}
\epsilon_{m}<\theta_{m, 0}<\mu_{m, 0}<\theta_{m, 1}<\cdots<\mu_{m, l}<\theta_{m, l+1}<\mu_{m, l+1}<\cdots<\delta_{m+1}, \\
\epsilon_{m}<\xi_{m, 0}<\mu_{m, 0}<\xi_{m, 1}<\cdots<\xi_{m, l}<\mu_{m, l}<\cdots<\delta_{m+1}, \\
\eta_{m+1}<\rho_{m, 0}<\tau_{m, 0}<\rho_{m, 1}<\tau_{m, 1}<\cdots<\rho_{m, l}<\tau_{m, l}<\cdots<\zeta_{m}, \\
\eta_{m+1}<\omega_{m, 0}<\tau_{m, 0}<\omega_{m, 1}<\cdots<\omega_{m, l}<\tau_{m, l}<\cdots<\zeta_{m} .
\end{gathered}
$$

It is easy to see that $\lim _{l \rightarrow \infty} \mu_{m, l}=\delta_{m+1}$, and $\lim _{l \rightarrow \infty} \tau_{m, l}=\zeta_{m}$.

Furthermore, we have the following results.

Proposition 2.11. Let $a<\lambda /\left(1-\lambda^{2}\right) \leq b<1 /(1-\lambda)$, and let $b \in\left(\epsilon_{m}, \delta_{m+1}\right)$ for $m \in N(1)$, then

(1) $\left(x_{n}, y_{n}\right) \rightarrow(1 /(1-\lambda), 0)$ as $n \rightarrow \infty$ if $\left(x_{0}, y_{0}\right) \in\left(x^{*}, \lambda b+1\right] \times\left(b, x^{*}\right]$;

(2) $\left(x_{n}, y_{n}\right) \rightarrow(0,1 /(1-\lambda))$ as $n \rightarrow \infty$ if $\left(x_{0}, y_{0}\right) \in\left(b, x^{*}\right] \times\left(x^{*}, \lambda b+1\right]$,

where $\epsilon_{m}$ and $\delta_{m+1}$ are given in Theorem 2.5, and $x^{*}$ is given in Theorem 2.8 .

Proposition 2.12. Let $a<0<b<\lambda /\left(1-\lambda^{2}\right)$ and let $b \in\left(\eta_{m+1}, \zeta_{m}\right)$ for $m \in N(1)$, then

(1) $\left(x_{n}, y_{n}\right) \rightarrow(1 /(1-\lambda), 0)$ as $n \rightarrow \infty$ if $\left(x_{0}, y_{0}\right) \in\left(\bar{x}^{*}, b / \lambda\right] \times\left(b, \bar{x}^{*}\right]$;

(2) $\left(x_{n}, y_{n}\right) \rightarrow(0,1 /(1-\lambda))$ as $n \rightarrow \infty$ if $\left(x_{0}, y_{0}\right) \in\left(b, \bar{x}^{*}\right] \times\left(\bar{x}^{*}, b / \lambda\right]$.

Here $\eta_{m+1}$ and $\zeta_{m}$ are given in Theorem 2.6, and $\bar{x}^{*}$ is given in Theorem 2.9.

Remark 2.13. It is easy to see that Theorems 2.5-2.9 and Propositions 2.3-2.12 are valid as $a=-\infty$.

\section{Proofs of main results}

By (1.1) and (1.2), it is easy to see that system (1.1) has an obvious connection with the following linear difference systems:

$$
\begin{array}{cccc}
x_{n+1}=\lambda x_{n}+1, & x_{n+1}=\lambda x_{n}+1, & x_{n+1}=\lambda x_{n}, & x_{n+1}=\lambda x_{n}, \\
y_{n+1}=\lambda y_{n}+1, & y_{n+1}=\lambda y_{n}, & y_{n+1}=\lambda y_{n}+1, & y_{n+1}=\lambda y_{n} .
\end{array}
$$

Therefore, we first consider the following relating equations:

$$
\begin{gathered}
u_{n+1}=\lambda u_{n}+1, \\
u_{n+1}=\lambda u_{n} .
\end{gathered}
$$

By induction, it is easy to check that, for $n \in N\left(n_{0}\right)$, the solution of (3.2) with the initial value $u_{n_{0}}=c$ is given by

$$
u_{n}=\lambda^{n-n_{0}} c+\frac{1-\lambda^{n-n_{0}}}{1-\lambda}, \quad n \in N\left(n_{0}+1\right),
$$


and the solution of (3.3) with the initial value $u_{n_{0}}=c$ is given by

$$
u_{n}=\lambda^{n-n_{0}} c, \quad n \in N\left(n_{0}+1\right) \text {. }
$$

Note that $\lambda \in(0,1)$, by formulations (3.4) and (3.5), it follows that $\lim _{n \rightarrow \infty} u_{n}=1 /(1-\lambda)$, and $\lim _{n \rightarrow \infty} u_{n}=0$, respectively.

By a direct iterative method, we can prove Propositions 2.1-2.12 and the following lemma.

Lemma 3.1. Let $a<0<b<1 /(1-\lambda)$. Then, for every solution $\left\{\left(x_{n}, y_{n}\right)\right\}$ of system (1.1) with the initial value $\left(x_{0}, y_{0}\right) \in \mathbb{R}^{2}$, there exists $a k \in \mathbb{N}$ such that one of the following results holds:

(1) $\left(x_{k}, y_{k}\right) \in I_{23}$

(2) $\left(x_{k}, y_{k}\right) \in I_{32}$;

(3) $\left(x_{k}, y_{k}\right) \in(b, \lambda b+1] \times(b, \lambda b+1] \cap(b, b / \lambda] \times(b, b / \lambda] \subseteq I_{33}$.

Now we give the proofs of our main results.

Proof of Theorem 2.5. By $\lambda /\left(1-\lambda^{2}\right) \leq b<1 /(1-\lambda)$, it follows that $\lambda b<b<\lambda b+1 \leq b / \lambda$. If $\left(x_{0}, y_{0}\right) \in(b, \lambda b+1] \times(b, \lambda b+1] \subseteq I_{33}$, then

$$
x_{1}=\lambda x_{0}<b, \quad y_{1}=\lambda y_{0}<b, \quad\left(x_{1}, y_{1}\right) \in(\lambda b, b] \times(\lambda b, b] \subseteq I_{22} .
$$

In view of Lemma 3.1, there exists $n_{1} \in \mathbb{N}$ such that

$$
\left(x_{n}, y_{n}\right) \in I_{22} \quad \text { for } n \in N\left(1, n_{1}\right), \quad\left(x_{n_{1}+1}, y_{n_{1}+1}\right) \notin I_{22},
$$

where

$$
x_{n_{1}}=\lambda^{n_{1}} x_{0}+\frac{1-\lambda^{n_{1}-1}}{1-\lambda} \leq b, \quad y_{n_{1}}=\lambda^{n_{1}} y_{0}+\frac{1-\lambda^{n_{1}-1}}{1-\lambda} \leq b .
$$

Since $b \in\left[\delta_{m}, \epsilon_{m}\right)$, we have

$$
\left(x_{m}, y_{m}\right) \in I_{22}, \quad\left(x_{m+1}, y_{m+1}\right) \in(b, \lambda b+1] \times(b, \lambda b+1] \subseteq I_{33},
$$

then $n_{1}=m$. For $l \in \mathbb{N}$ and $k \in N(1, m)$, repeating the above proceeding, we have

$$
\left(x_{(m+1) l}, y_{(m+1) l}\right) \in(b, \lambda b+1] \times(b, \lambda b+1], \quad\left(x_{(m+1) l+k}, y_{(m+1) l+k}\right) \in I_{22} .
$$

In terms of (3.2) and (3.3), we define

$$
f_{1}(x)=\lambda x+1, \quad f_{2}(x)=\lambda x,
$$

and for $(x, y) \in(b, \lambda b+1] \times(b, \lambda b+1]$, we define

$$
P_{m+1}(x)=\left(f_{1}^{(m)} \circ f_{2}\right)(x), \quad R_{m+1}(x, y)=\left(P_{m+1}(x), P_{m+1}(y)\right), \quad R_{m+1}^{(n+1)}=R_{m+1} \circ R_{m+1}^{(n)} \text {. }
$$


It follows that

$$
\begin{gathered}
R_{m+1}(x, y)=\left(\lambda^{m+1} x+\frac{1-\lambda^{m}}{1-\lambda}, \lambda^{m+1} y+\frac{1-\lambda^{m}}{1-\lambda}\right), \\
R_{m+1}^{(n)}(x, y)=\left(\lambda^{n(m+1)} x+\frac{1-\lambda^{m}}{1-\lambda} \cdot \frac{1-\lambda^{n(m+1)}}{1-\lambda^{m+1}}, \lambda^{n(m+1)} y+\frac{1-\lambda^{m}}{1-\lambda} \cdot \frac{1-\lambda^{n(m+1)}}{1-\lambda^{m+1}}\right),
\end{gathered}
$$

and $\lim _{n \rightarrow \infty} R_{m+1}^{(n)}(x, y)=\left(\epsilon_{m}, \epsilon_{m}\right)$.

In fact, $\left(\epsilon_{m}, \epsilon_{m}\right)$ is the unique fixed point of $R_{m+1}(x, y)$, and the solution $\left\{\left(\bar{x}_{n}, \bar{y}_{n}\right)\right\}$ of system (1.1) with the initial value $\left(\epsilon_{m}, \epsilon_{m}\right)$ is periodic with minimal period $m+1$. By (3.13), it follows that

$$
\left(x_{(m+1) l}, y_{(m+1) l}\right)=R_{m+1}^{(l)}\left(x_{0}, y_{0}\right) \quad \text { for }\left(x_{0}, y_{0}\right) \in(b, \lambda b+1] \times(b, \lambda b+1] .
$$

Therefore for any solution $\left\{\left(x_{n}, y_{n}\right)\right\}$ of system (1.1) with the initial value $\left(x_{0}, y_{0}\right) \in$ $(b, \lambda b+1] \times(b, \lambda b+1]$, we can get $\lim _{n \rightarrow \infty}\left(x_{n}-\bar{x}_{n}\right)=\lim _{n \rightarrow \infty}\left(y_{n}-\bar{y}_{n}\right)=0$. The proof is complete.

Proof of Theorem 2.6. By $0<b<\lambda /\left(1-\lambda^{2}\right)$, we have $(b-1) / \lambda<\lambda b<b<b / \lambda<\lambda b+1$. If $\left(x_{0}, y_{0}\right) \in(b, b / \lambda] \times(b, b / \lambda] \subseteq I_{33}$, then $x_{1}=\lambda x_{0}, y_{1}=\lambda y_{0}, x_{2}=\lambda^{2} x_{0}+1, y_{2}=\lambda^{2} y_{0}+1$, where $\left(x_{1}, y_{1}\right) \in I_{22},\left(x_{2}, y_{2}\right) \in(b, \lambda b+1] \times(b, \lambda b+1] \subseteq I_{33}$, and

$$
x_{n}=\lambda^{n-2} x_{2}=\lambda^{n} x_{0}+\lambda^{n-2}, \quad y_{n}=\lambda^{n-2} y_{2}=\lambda^{n} y_{0}+\lambda^{n-2}, \quad n \in N(2) .
$$

Since $b \in\left[\zeta_{m}, \eta_{m}\right)$, we have

$$
\begin{gathered}
\left(x_{n}, y_{n}\right) \in\left(\frac{b}{\lambda}, \lambda b+1\right] \times\left(\frac{b}{\lambda}, \lambda b+1\right], \quad n \in N(2, m), \\
\left(x_{m+1}, y_{m+1}\right) \in\left(b, \frac{b}{\lambda}\right] \times\left(b, \frac{b}{\lambda}\right], \quad\left(x_{m+2}, y_{m+2}\right) \in I_{22}, \quad m \in N(2) .
\end{gathered}
$$

For $l \in \mathbb{N}$, repeating the above proceeding, it follows that

$$
\begin{gathered}
\left(x_{(m+1) l+k}, y_{(m+1) l+k}\right) \in\left(\frac{b}{\lambda}, \lambda b+1\right] \times\left(\frac{b}{\lambda}, \lambda b+1\right], \quad k \in N(2, m), \\
\left(x_{(m+1) l+1}, y_{(m+1) l+1}\right) \in I_{22}, \quad\left(x_{(m+1) l}, y_{(m+1) l}\right) \in\left(b, \frac{b}{\lambda}\right] \times\left(b, \frac{b}{\lambda}\right] .
\end{gathered}
$$

In view of (3.11), for $(x, y) \in(b, b / \lambda] \times(b, b / \lambda]$, we define

$$
G_{p+1}(x, y)=\left(f_{2}^{(p-1)} \circ f_{1} \circ f_{2}(x), f_{2}^{(p-1)} \circ f_{1} \circ f_{2}(y)\right),
$$


Honghua Bin et al. 9

and set $G_{p+1}^{(n+1)}=G_{p+1} \circ G_{p+1}^{(n)}$. Thus, we have

$$
\begin{gathered}
G_{p+1}(x, y)=\left(\lambda^{p+1} x+\lambda^{p-1}, \lambda^{p+1} y+\lambda^{p-1}\right) \\
G_{p+1}^{(n)}(x, y)=\left(\lambda^{n(p+1)} x+\frac{\lambda^{p-1}\left(1-\lambda^{n(p+1)}\right)}{1-\lambda^{p+1}}, \lambda^{n(p+1)} y+\frac{\lambda^{p-1}\left(1-\lambda^{n(p+1)}\right)}{1-\lambda^{p+1}}\right),
\end{gathered}
$$

and $\lim _{n \rightarrow \infty} G_{m+1}^{(n)}(x, y)=\left(\eta_{m}, \eta_{m}\right)$. In view of (3.19), for $\left(x_{0}, y_{0}\right) \in(b, b / \lambda] \times(b, b / \lambda]$, we have $\left(x_{(m+1) l}, y_{(m+1) l}\right)=G_{m+1}^{(l)}\left(x_{0}, y_{0}\right)$.

Obviously, $\left(\eta_{m}, \eta_{m}\right)$ is the unique fixed point of $G_{m+1}$ and the solution $\left\{\left(\bar{x}_{n}, \bar{y}_{n}\right)\right\}$ of system (1.1) with the initial value $\left(\eta_{m}, \eta_{m}\right)$ is periodic with minimal period $m+1$. Moreover, for any solution $\left\{\left(x_{n}, y_{n}\right)\right\}$ of system $(1.1)$ with the initial value $\left(x_{0}, y_{0}\right) \in(b, b / \lambda] \times$ $(b, b / \lambda]$, we have $\lim _{n \rightarrow \infty}\left(x_{n}-\bar{x}_{n}\right)=\lim _{n \rightarrow \infty}\left(y_{n}-\bar{y}_{n}\right)=0$. The proof is complete.

Proof of Theorem 2.8. We only prove the first claim, the other is similar.

For $x \in(b, \lambda b+1]$, we set $P_{m+1}(x)=\left(f_{1}^{(m)} \circ f_{2}\right)(x)$, where $f_{1}$ and $f_{2}$ have been given in (3.11), and we have

$$
P_{m+1}(x)=\lambda^{m+1} x+\frac{1-\lambda^{m}}{1-\lambda}, \quad m \in N(1)
$$

Note $b \in\left(\epsilon_{m}, \delta_{m+1}\right)$, we have $0<x<1 /(1-\lambda), P_{m}(x)<P_{m+1}(x)$, and $P_{m+1}\left(x^{*}\right)=b$, $P_{m+2}\left(x^{*}\right)=\lambda b+1$. Moreover $P_{m+1}(x) \in(b, \lambda b+1]$ for $x \in\left(x^{*}, \lambda b+1\right]$, and $P_{m+2}(x) \in$ $(b, \lambda b+1]$ for $x \in\left(b, x^{*}\right]$.

Since $b \geq \theta_{m, 0}$, we have

$$
P_{m+1}(\lambda b+1) \leq x^{*}, \quad P_{m+1}\left(\left(x^{*}, \lambda b+1\right]\right) \subseteq\left(b, x^{*}\right] .
$$

Furthermore, by $b \in\left[\theta_{m, l}, \mu_{m, l}\right)$, it follows that

$$
P_{m+2}^{(l)} \circ P_{m+1}(\lambda b+1) \leq x^{*}, \quad P_{m+2}^{(l+1)} \circ P_{m+1}\left(x^{*}\right)>x^{*} .
$$

If the initial value $\left(x_{0}, y_{0}\right) \in\left(x^{*}, \lambda b+1\right] \times\left(x^{*}, \lambda b+1\right]$, then, for $b \in\left[\theta_{m, l}, \mu_{m, l}\right)$ and $n \in$ $N(1)$, we have

$$
\begin{gathered}
\left(x_{m+1+(m+2) n}, y_{m+1+(m+2) n}\right)=\left(P_{m+2}^{(n)} \circ P_{m+1}\left(x_{0}\right), P_{m+2}^{(n)} \circ P_{m+1}\left(y_{0}\right)\right), \\
\left(x_{m+1+(m+2) k}, y_{m+1+(m+2) k}\right) \in\left(b, x^{*}\right] \times\left(b, x^{*}\right] \quad \text { for } k \in N(0, l),
\end{gathered}
$$

and $\left(x_{m+1+(m+2)(l+1)}, y_{m+1+(m+2)(l+1)}\right) \in\left(x^{*}, \lambda b+1\right] \times\left(x^{*}, \lambda b+1\right]$.

In view of (3.22), for $(x, y) \in\left(x^{*}, \lambda b+1\right] \times\left(x^{*}, \lambda b+1\right]$, we denote

$$
H(x, y)=\left(P_{m+2}^{(l+1)} \circ P_{m+1}(x), P_{m+2}^{(l+1)} \circ P_{m+1}(y)\right),
$$

and it follows that $\left(x_{(m+2)(l+2)-1}, y_{(m+2)(l+2)-1}\right)=H\left(x_{0}, y_{0}\right)$.

Obviously, there exists a $\left(\bar{x}_{0}, \bar{y}_{0}\right) \in\left(x^{*}, \lambda b+1\right] \times\left(x^{*}, \lambda b+1\right]$ such that

$$
\lim _{n \rightarrow \infty} H^{(n)}(x, y)=\left(\bar{x}_{0}, \bar{y}_{0}\right) \quad \text { for }(x, y) \in\left(x^{*}, \lambda b+1\right] \times\left(x^{*}, \lambda b+1\right],
$$


where $\left(\bar{x}_{0}, \bar{y}_{0}\right)$ is the unique fixed point of $H$. Therefore, the solution $\left\{\left(\bar{x}_{n}, \bar{y}_{n}\right)\right\}$ of system (1.1) with the initial value $\left(\bar{x}_{0}, \bar{y}_{0}\right) \in\left(x^{*}, \lambda b+1\right] \times\left(x^{*}, \lambda b+1\right]$ is periodic with minimal period $(m+2)(l+2)-1$. Moreover, for any solution $\left\{\left(x_{n}, y_{n}\right)\right\}$ of system (1.1) with the initial value $\left(x_{0}, y_{0}\right) \in\left(x^{*}, \lambda b+1\right] \times\left(x^{*}, \lambda b+1\right]$, we have $\lim _{n \rightarrow \infty}\left(x_{n}-\bar{x}_{n}\right)=\lim _{n \rightarrow \infty}\left(y_{n}-\right.$ $\left.\bar{y}_{n}\right)=0$. The proof is complete.

Proof of Theorem 2.9 is similar to that of Theorem 2.8 and is omitted.

\section{Acknowledgment}

This project is supported by Yuyan Foundation of Jimei University.

\section{References}

[1] R. P. Agarwal, Difference Equations and Inequalities. Theory, Methods, and Applications, 2nd ed., Monographs and Textbooks in Pure and Applied Mathematics, vol. 228, Marcel Dekker, New York, 2000.

[2] Y. Chen and J. Wu, Minimal instability and unstable set of a phase-locked periodic orbit in a delayed neural network, Physica D 134 (1999), no. 2, 185-199.

[3] Y. Chen, J. Wu, and T. Krisztin, Connecting orbits from synchronous periodic solutions in phaselocked periodic solutions in a delay differential system, Journal of Differential Equations $\mathbf{1 6 3}$ (2000), no. 1, 130-173.

[4] A. Destexhe and P. Gaspard, Bursting oscillations from a homoclinic tangency in a time delay system, Physics Letters A 173 (1993), no. 4-5, 386-391.

[5] K. Gopalsamy and I. Leung, Delay induced periodicity in a neural netlet of excitation and inhibition, Physica D 89 (1996), no. 3-4, 395-426.

[6] J. J. Hopfield, Neurons with graded response have collective computational properties like those of two-state neurons, Proceedings of the National Academy of Sciences of the United States of America 81 (1984), no. 10, 3088-3092.

[7] L. Huang and J. Wu, Dynamics of inhibitory artificial neural networks with threshold nonlinearity, Fields Institute Communications 29 (2001), 235-243.

[8] W. G. Kelley and A. C. Peterson, Difference Equations: An Introduction with Applications, Academic Press, Massachusetts, 1991.

[9] Y. M. Meng, L. Huang, and K. Y. Liu, Asymptotic behavior of solutions for a class of neural network models of two neurons with two thresholds, Acta Mathematicae Applicatae Sinica 26 (2003), no. 1, $158-175$.

[10] Z. Yuan, L. Huang, and Y. Chen, Convergence and periodicity of solutions for a discrete-time network model of two neurons, Mathematical and Computer Modelling 35 (2002), no. 9-10, 941950.

Honghua Bin: School of Sciences, Jimei University, Xiamen, Fujian 361021, China

E-mail address: binhonghua@163.com

Lihong Huang: College of Mathematics and Econometrics, Hunan University, Changsha,

Hunan 410082, China

E-mail address: 1hhuang@hnu.net.cn

Guang Zhang: Department of Mathematics, Qingdao Institute of Architecture and Engineering,

Qingdao, Shandong 266033, China

E-mail address: dtgzhang@yahoo.com.cn 